\title{
Características Estructurales y Fisiológicas de Petunia axillaris ReLACIONADAS CON SU BAJA SENSIBILIDAD A GLIFOSATO ${ }^{1}$
}

\author{
Structural and Physiological Characteristics of Petunia axillaris Related to their Low Sensitivity \\ to Glyphosate
}

\author{
DELLAFERRERA, I..$^{2,3}$ PANIGO, E. ${ }^{2}$, GONZALEZ-TORRALBA, F. ${ }^{4}$, DE PRADO, R. ${ }^{4}$, \\ CHRISTOFFOLETI, P. ${ }^{4}$ y PERRETA, M. ${ }^{2}$
}

\begin{abstract}
RESUMEN - La utilización repetida de glifosato como herbicida, entre otros aspectos, aumenta la frecuencia de individuos con baja sensibilidad al mismo. Entre los numerosos casos reportados de especies de malezas que han presentado algún inconveniente para su adecuado manejo se encuentra Petunia axillaris. En este trabajo se evalúa su sensibilidad y se estudian posibles mecanismos involucrados. Se analizó la retención, absorción y translocación con herbicida radiomarcado, así como también la modificación estructural producida por la aplicación del herbicida. Los análisis comprueban que Petunia axillaris es una especie tolerante a glifosato, no mostrando inhibición de la ruta metabólica del ácido shikímico. La menor sensibilidad al herbicida podría estar relacionada con una menor retención foliar de glifosato y un mayor tiempo de absorción; lo cual retarda el ingreso y movimiento del herbicida. Desde el punto de vista estructural se afecta el desarrollo del eje principal, mateniendo sin embargo una elevada producción de flores y semillas. Petunia axillaris muestra una combinación de características morfológicas-estructurales y fisiológicas que determinan su ventaja adaptativa para permanecer y aumentar su población en sistemas con uso intensivo de glifosato.
\end{abstract}

Palabras-clave: malezas, tolerancia, forma de crecimiento, capacidad reproductiva.

\begin{abstract}
Repeated use of glyphosate as herbicide in the same site, among other things, increases the frequency of individuals with low sensitivity. Petunia axillaris is among the many reported cases of weed species that have presented problems for proper management. This study evaluates their sensitivity and discusses the possible mechanisms involved in its differential sensitivity. For this purpose, we analyzed the retention, absorption and translocation of radiolabeled herbicide, and the morphological characteristics related to the herbicide response. Studies confirms that Petunia axillaris is a glyphosate tolerant specie and the shikimic acid metabolic pathway is not altered. The low sensitivity to glyphosate in Petunia axillaris could be associated to low foliar retention and increased time of absorption, which delayed the entrance and movement of the herbicide. Regardless the altered structure with glyphosate, seed production is high enough which ensures the perpetuation of the less sensitive genotypes. Petunia axillaris showed a combination of morphological, structural, and physiological characteristics that determined their adaptive advantage to perpetuate and increase its population in systems with intensive use of glyphosate.
\end{abstract}

Keywords: weeds, tolerance, growth form, reproductive capacity.

1 Recebido para publicação em 27.11.2014 e aprovado em 27.4.2015.

2 Facultad de Ciencias Agrarias - Universidad Nacional del Litoral, Esperanza-Santa Fe, Argentina, <idellaferrera@gmail.com>; ${ }^{3}$ Departamento de Química Agrícola y Edafología - Universidad de Córdoba - Córdoba - Andalucía - España; ${ }^{4}$ Escola Superior de Agricultura “Luiz de Queiroz” - Universidade de São Paulo - Piracicaba - São Paulo - Brasil. 


\section{INTRODUCCIÓN}

Una consecuencia del uso intensivo de un herbicida con el mismo ingrediente activo durante un largo período de tiempo es la aparición de malezas que muestran algún grado de tolerancia o resistencia (Christoffoleti et al., 2008) o mecanismos que les permiten escapar de la acción del herbicida (Scursoni $\&$ Satorre, 2010). Numerosas especies han sido reportadas como tolerantes a glifosato, sin embargo, las causas de esta sensibilidad diferencial no es aun completamente comprendida y la información disponible es limitada (Owen, 2008).

Petunia axillaris es una de las especies que muestra problemas en cuando a su manejo y se la ha descrito como invasora de sistemas de cultivo de soja en siembra directa con aplicaciones intensivas de glifosato (Puricelli \& Papa, 2006; Dellaferrera et al., 2007). Esta especie muestra una alta tolerancia, reportándose en estudios de campo su supervivencia a dosis de $51^{*}$ ha-1 de glifosato formulado al 48\% p/v (Trejo \& Sobrero, 2012).

La resistencia a glifosato puede ser resultado de: mutaciones en el DNA (Powles \& Yu, 2010), sobreexpresión de la enzima EPSPS (Gaines et al., 2011), tazas de absorción o translocación reducida (Feng et al., 2004), secuestración del herbicida en vacuolas (Ge et al., 2010), alteración de la enzima EPSPS (Baerson et al., 2002) o variaciones en el metabolismo del glifosato (Rojano-Delgado et al., 2012). Asociado a la baja eficacia del glifosato, independientemente de sus causas, entran en juego otros factores que determinan que las especies o los genotipos de una especie que sobreviven, se reproduzcan y aumenten su frecuencia dentro de una determinada área, resultando en un cambio en la composición y abundancia de poblaciones de malezas (Johnson et al., 2009).

Cuando una planta es expuesta a la acción de un herbicida y sobrevive, puede modificar su estructura o comportamiento (Meusel et al., 1977), debido principalmente a la acción ejercida por el herbicida sobre los meristemas. Bajo condiciones óptimas de crecimiento, sin perturbaciones, el desarrollo de la planta refleja estrictamente su arquitectura, pero ante factores estresantes puede desviarse de ella, modificando su fisiología y redirigiendo el crecimiento (Malpassi, 2004). Esto último se verá reflejado en la estructura de la planta dado que la arquitectura no es más que la resultante del equilibrio entre los procesos endógenos de crecimiento y las acciones exteriores ejercidas por el ambiente en un determinado momento (Barthélémy et al., 1989). La posibilidad de continuar el crecimiento o rebrotar desde zonas no dañadas permite a los biotipos menos sensibles aumentar su densidad en la población, si las condiciones de cultivo no se modifican. Muchas especies anuales como Conyza bonariensis (Mellendorf et al., 2013) y Poa annua (Binkholder et al., 2011) presentan un mecanismo de rebrote que les asegura la producción de semilla.

Actualmente, no se cuenta con estudios de mecanismos tolerancia, ni estructurales para $P$. axilaris. Por lo que los objetivos de este trabajo son: (a) evaluar la sensibilidad de $P$. axilaris a glifosato y la acumulación de ácido shikimico; (b) determinar la capacidad de retención foliar, absorción y translocación del herbicida; y (c) evaluar su estrategia de brotación y sus modificaciones estructurales luego que la planta sobrevive a la acción del herbicida.

\section{MATERIALES Y METODOS}

\section{Material vegetal y condiciones de cultivo}

Se recolectaron semillas de $P$. axillaris y A. hybridus ssp hybridus de plantas maduras en la localidad de Esperanza, Argentina

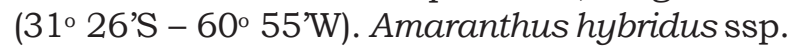
hybridus es una maleza común que ha reducido su abundancia relativa debido al sistema actual de cultivo en Argentina y su conocida susceptibilidad al herbicida glifosato, por tanto puede ser usada como patrón para comparar la sensibilidad relativa de otras especies (Cruz-Hipolito et al., 2011; RojanoDelgado et al., 2012).

Las semillas se pusieron a germinar en placas de Petri sobre papel de filtro húmedo. Las plántulas fueron luego transplantadas a macetas individuales que contenian una mezcla de arena y limo $(1: 2 \mathrm{v} / \mathrm{v})$ y puestas en 
sala de crecimiento a temperatura alternada de $28 / 18{ }^{\text {a }} \mathrm{C}$ dia/noche con un fotoperiodo de 16 hs y una intensidad luminica de $350 \mu \mathrm{mol} \mathrm{m} \mathrm{m}^{-2} \mathrm{~s}^{-1}$ y un $80 \%$ de humedad relativa. Las plantas se regaron a diario y no se usó fertilizante.

\section{Ensayos de dosis - respuesta}

Cuando las plantas alcanzaron las 3 a 4 hojas totalmente desarolladas, estado 1,3 a 1,4 según la escala fenológica $\mathrm{BBCH}$ (Hess et al., 1997) se aplicó glifosato (Roundup ${ }^{\circledR}$ Power MAX como sal isopropylamina, $48 \% \mathrm{p} / \mathrm{v}$ ) en dosis de $0,25,50,100,200$ y 400 g a.e. ha ${ }^{-1}$ para $A$. hybridus ssp. hybridus y 0, 100, 200, 400,800 y 1.600 g a.e. ha-1 para $P$. axillaris. Los tratamientos se realizaron con una cámara de aplicación de laboratorio con pastillas de abanico plano calibradas para erogar $200 \mathrm{~L} \mathrm{ha}^{-1}$ a $275 \mathrm{kPa}$.

Se determinó el peso fresco por planta a los 21 dias post aplicación y los resultados fueron expresados como porcentaje del testigo sin tratar. Los datos fueron ajustados a un modelo de regresión logístico no lineal de tres parámetros (ec. 1) con la asintota superior fijada en $100 \%$; se utilizó el software estadístico "R" con "drc plug-in" (Ritz \& Streibig, 2005).

$$
f(x)=c+\frac{d-c}{1+\exp b(\log (x)-\log (E D 50))}
$$

En esta ecuación $E D 50$ es la dosis que produce la respuesta media entre el límite superior $d$ (fijado en $100 \%$ ) y el límite inferior $c$, el parámetro $b$ muestra la pendiente alrededor de ED50 (Streibig \& Kudsk, 1993). La dosis de herbicida para reducir el peso un $50 \%$ respecto al testigo sin tratar se determinó para cada especie. Mediante el test de $t$ Student $(p>0,05)$ se determinó la significancia del nivel de tolerancia que fue calculado como (ED50 P. axillaris)/ED50 (A. hybridus). Los tratamientos siguieron un diseño completamente al azar con diez repeticiones por especie, el experimento completo fue repetido tres veces.

\section{Acumulación de ácido shikímico}

Plantas de $P$. axillaris y A. hybridus ssp. hybridus fueron tratadas con glifosato a una dosis de $100 \mathrm{~g}$ a.i. ha como se describió más arriba. Se cosecharon hojas de las plantas de ambas especies en estado 1,3 a 1,4 según (Hess et al., 1997) para la determinación de ácido shikímico a 24, 48, 72, y 96 horas post tratamiento (HPT). Los tejidos foliares $(50 \mathrm{mg}$ de peso fresco) fueron homogenizados y congelados a $-40{ }^{\circ} \mathrm{C}$ siguiendo el protocolo de Singh \& Shaner (1998). La acumulación de ácido shikímico se determinó ópticamente a $380 \mathrm{~nm}$ con un espectrofotómetro Beckman DU-640. La curva estándar de calibración se determinó utilizando una escala de concentración de ácido conocida. Los valores se expresaron como acumulación por sobre los valores de plantas testigo sin tratar de cada especie. Los tratamientos siguieron un diseño completamente al azar con diez repeticiones por especie y por tiempo, el experimento completo fue repetido tres veces.

\section{Retención foliar de herbicida}

Se siguió la metodología descrita por Gauvrit (2003). Un grupo de plantas de A. hybridus ssp. hybridus y $P$. axillaris al estado $\mathrm{BBCH} 1.3$ to 1.4 según (Hess et al., 1997) fueron asperjadas con una solución coloreada de glifosato $100 \mathrm{~g}$ a.i. ha ${ }^{-1} \mathrm{y}$ $100 \mathrm{mg} \mathrm{L}^{-1}$ de Na-fluoresceina en un volumen equivalente de $200 \mathrm{~L}$ de agua. Luego de que la solución se secara sobre las hojas las plantas fueron cosechadas a nivel del suelo y sumergidas por 30 s en $50 \mathrm{~mL}$ de $5 \mathrm{mM} \mathrm{NaOH}$. El lavado fue leído en espectrofluorímetro a $490 / 510 \mathrm{~nm}$. Las plantas fueron secadas a $65^{\circ} \mathrm{C}$ for $72 \mathrm{~h}$, para determinar el peso seco. Luego la retención de herbicida fue expresada en función del peso seco. Se utilizaron diez repeticiones por tratamiento (tres plantas por tratamiento). Se utilizó el test de t de Student $(p>0,05)$ para evaluar diferencias respecto a la especie.

\section{Absorcion y translocación de $\left[{ }^{14} \mathrm{C}\right]$ glifosato}

Para las determinaciones el $\left[{ }^{14} \mathrm{C}\right]$ glifosato fue mezclado con glifosato formulado comercialmente para preparar una solución con una actividad específica de $0.834 \mathrm{kBq} \mu \mathrm{L}^{-1}$ y una concentración de glifosato de $0.5 \mathrm{~g}$ a.i. $\mathrm{L}^{-1}$ (equivalente a $100 \mathrm{~g}$ a.i. ha-1 en $200 \mathrm{~L}$ ). Se utilizaron plantas de ambas especies. E1 herbicida radiomarcado fue aplicado en gotas 
de $1 \mu \mathrm{L}$ en la cara adaxial de la última hoja totalmente expandida utilizando una micropipeta. Se cosecharon plantas a 24,48 , 72 y 96 h post aplicación. Se separó la hoja tratada y el herbicida no absorbido fue removido sumergiendo a esta en $3 \mathrm{~mL}$ de una solución de acetona-agua $(1: 1 \mathrm{v} / \mathrm{v})$. Las plantas fueron seccionadas en hoja tratada y resto de la planta. El lavado fue mezclado con $12 \mathrm{~mL}$ de líquido de centelleo y analizado con un contador de centelleo líquido (LSS) (Scintillation Counter, Beckman LS 6500). Cada porción de tejido se secó a $65^{\circ} \mathrm{C}$ por $72 \mathrm{~h}$ y fue quemada en un oxidador biológico (Packard Tri Carb 307). $\mathrm{El}^{14} \mathrm{CO}_{2}$ generado efue contenido en $18 \mathrm{~mL}$ de una mezcla de CarboSorb E y Permafluor (1:1 v/v) (Perkin-Elmer, Packard Bioscience BV). La radioactividad fue cuantificada mediante LSS. Se realizaron 5 repeticiones por especie y por tiempo de muestreo.

La absorción se cuantificó por cada tiempo de muestreo y por especie como $[\mathrm{kBq}$ resto de la planta / $(\mathrm{kBq}$ resto de la planta $+\mathrm{kBq}$ en lavado)*100]. Los datos fueron analizados siguiendo la metodología propuesta por Kniss et al., (2011) ajustándolos a una regresión hiperbólica cuadrática (ec. 2), usando "R" con “drc plug-in" (Ritz \& Streibig, 2005).

$$
\text { Absorción } n=\frac{A \max * t}{0,11 * t 90+t}
$$

Donde Amax representa la asíntota de máxima absorción, $t$ es el tiempo post aplicación y $t 90$ el tiempo en alcanzar el 90\% de Amax. Los parámetros Amax y $t 90$ fueron comparados mediante el test de $t$ de Student para evaluar diferencias debidas a la especie.

La translocación se cuantificó como [(kBq en resto de la planta) / ( $\mathrm{kBq}$ en resto de la planta $+\mathrm{kBq}$ en hoja tratada)*100]. Los datos fueron ajustados a un modelo Gompertz dado por la ec. 3 utilizando el mismo software estadístico que en absorción.

$$
\text { Translocación }=\operatorname{Tmax} * e^{-\exp -\frac{t-k}{b}}
$$

Siendo Tmax la asíntota de máxima translocación, $t$ es el tiempo post aplicación, $k$ es el punto de inflexión y $b$ es la pendiente en k. Se comparó el parámetro Tmax para evaluar diferencias debidas a la especie.

\section{Autoradiografia}

Se utilizó un "phosphor imager cyclone" (Perkin-Elmer, Packard Bioscience BV) para visualizar el patrón de translocación del herbicida. Las plantas de A. hybridus ssp. hybridus y $P$. axillaris previamente tratadas con herbicida marcado y no marcado como se describe en los párrafos previos fueron cosechadas a los distintos tiempos propuestos para los ensayos de absorción y translocación luego prensadas y secadas a $50{ }^{\circ} \mathrm{C}$ por 4 dias. Las plantas secas fueron colocadas en film sensible por $4 \mathrm{~h}$ y escaneadas para determinar la dispersión del herbicida marcado en los tejidos.

\section{Forma de crecimiento}

Para evaluar el efecto de la aplicación de glifosato sobre la forma de crecimiento se aplicó una dosis de 960 g i.a. ha ${ }^{-1}$ sobre un grupo de 20 plantas y se mantuvo un grupo de control, las plantas crecieron en las condiciones de cámara descritas al inicio de esta sección. La aplicación se realizó cuando la planta presentaba entre 9 y 10 hojas expandidas, en estado vegetativo (estructura en forma de roseta). Diariamente, luego de la aplicación, se observó y registró el estado de las plantas y la aparición de síntomas. Finalmente, las plantas sobrevivientes fueron diseccionadas para determinar su estructura a los 90 días de emergencia.

Para lograr una completa caracterización de la estructura se trabajó además con material coleccionado a campo en distintos estados de desarrollo, el que fue previamente herborizado y posteriormente analizado bajo microscopio estereoscópico.

\section{RESULTADOS Y DISCUSIÓN}

Petunia axillaris forma parte de una nueva comunidad de malezas asociadas al cultivo de soja con aplicaciones intensivas de glifosato (Faccini \& Puricelli, 2007). La causa principal de estos cambios en las comunidades es que el glifosato, al igual que otros herbicidas, cuando es utilizado por largos períodos se 
transforma en un factor de selección (Galon et al., 2013), modificando fuertemente el agroecosistema y favoreciendo la aparición de especies y biotipos tolerantes (Christoffoleti et al., 2008; Galon et al., 2013).

Los ensayos de dosis-respuestas mostraron que $P$. axillaris presenta una menor sensibilidad que $A$. hybridus ssp. hybridus (Figura 1). Al incrementar la dosis de glifosato la biomasa de ambas especies se reduce. Sin embargo, la dosis requerida para reducir un $50 \%$ el peso fresco (ED50) es 3,7 veces mayor en $P$. axillaris; mostrando una mayor tolerancia al glifosato $\left(\mathrm{ED}_{50}=116,38 \mathrm{~g}\right.$ a.i. $\left.\mathrm{ha}^{-1}\right)$. En otros trabajos que utilizaron la misma especie sensible como patrón, se encontraron factores de tolerancia similares a este trabajo; con un rango que va entre 2,5 y 14,2 (Cruz-Hipolito et al., 2011; Rojano-Delgado et al., 2012).

El glifosato, entre otros efectos, actúa inhibiendo la EPSPs, la inhibición de esta enzima en la ruta del shikimato tiene como consecuencia la acumulación de ácido shikímico; y esta respuesta puede usarse como indicador de la sensibilidad de una especie a la exposición del glifosato (Velini et al., 2008). En este trabajo luego del tratamiento con glifosato los niveles de ácido shikimico acumulados, por encima del testigo sin tratar, fueron significativamente mayores en A. hybridus ssp. hybridus que en $P$. axillaris, para todos los tiempos medidos
(Figura 2). Estos niveles alcanzaron valores de 0,15 y $2,61\left(\mathrm{mg} \mathrm{g}^{-1}\right.$ hoja) en $P$. axillaris y A. hybrids respectivamente a las 96 HAT. Esto muestra que no hay inhibición de la EPSPs en $P$. axillaris. Además el hecho de $P$. axillaris presente altos valores de $\mathrm{ED}_{50} \mathrm{y}$ bajas concentraciones de ácido shikímico permite inferir que la ruta del shikimato no se encuentra alterada por la aplicación de glifosato, como ya fue demostrado para otras especies tolerantes o resistentes. (Feng et al., 2004).

La eficacia del glifosato depende de la retención del producto sobre la hoja, de la absorción por vía foliar del ingrediente activo y finalmente de su translocación al resto de la planta hasta el sitio de acción. Con respecto a la retención, ambas especies mostraron valores altos (Tabla 1), aunque significativamente distintos y mayores para A. hybridus ssp. hybridus. Petunia axillaris retuvo 1,4 veces menos herbicida que A. hybridus ssp. hybridus, hecho que podria estar relacionado a la baja sensibilidad encontrada en la especie. Se ha descrito que las alteraciones en la retención foliar constituyen mecanismos potenciales de resistencia (Feng et al., 2004); en el género Conyza la menor sensibilidad encontrada, fue correlacionada con una baja retención foliar (Gonzalez-Torralva et al., 2010).

En cuanto a la absorción los modelos ajustados muestran correlación con los

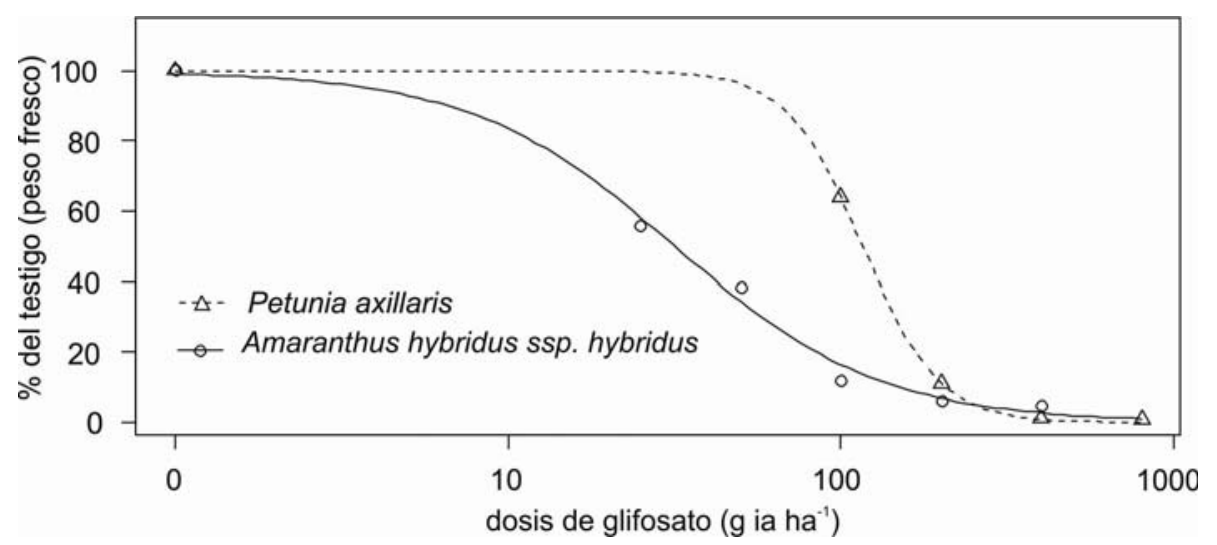

Cada punto es la media de tres experimentos. Estas curvas fueron calculadas con el modelo logístico no lineal de tres parámetros.

Figura 1 - Curvas de dosis respuesta correspondientes a P. axillaris y A. hybridus ssp. hybridus luego de la aplicación de glifosato en dosis crecientes. El peso fresco de la parte aérea de las plantas fue determinado a los 21 días post aplicación de glifosato y está expresado como porcentaje del testigo sin tratar. 


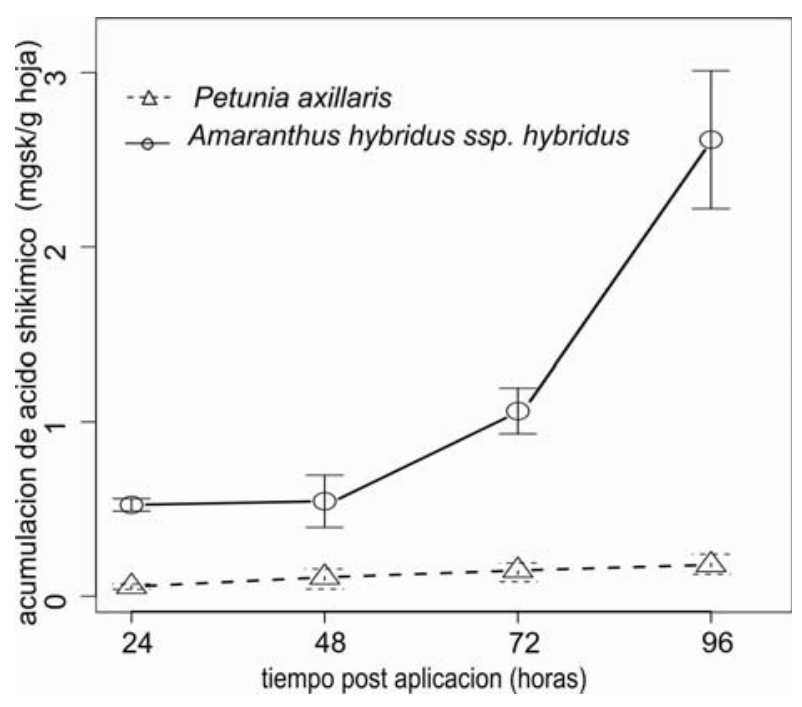

Figura 2 - Ácido shikimico acumulado en hojas de plantas de P. axillaris y A. hybridus ssp. hybridus en estado $\mathrm{BBCH}$ 1.3 luego de una aplicación de $100 \mathrm{~g}$ i.a. ha ${ }^{-1}$ de glifosato. Las barras verticales representan los errores estándar de las medias.

Tabla 1 - Retención foliar de glifosato en A. hybridus ssp. hybridus y Petunia axillaris. Los valores están expresados en $\mathrm{mL}$ de herbicida por $\mathrm{g}$ de peso seco

\begin{tabular}{|l|c|}
\hline \multicolumn{1}{|c|}{ Especie } & $\begin{array}{c}\text { Media (DE) } \\
\left(\mathrm{mL}^{*} \mathrm{~g}^{-1}\right)\end{array}$ \\
\hline A. hybridus ssp. hybridus & $0,7031(0.1727)$ \\
\hline Petunia axillaris & $0,5010^{*}(0,0349)$ \\
\hline
\end{tabular}

Medias seguidas por asterisco difieren significativamente según test de $\mathrm{t}$ de Student $(a ́=0,05)$.

valores medidos (Tabla 2). El valor estimado de absorción máxima (Amax) no es significativamente distinto entre especies, aunque el tiempo en alcanzar el $90 \%$ de este valor (t90) varió considerablemente entre especies. La máxima absorción en $A$. hybridus ssp. hybridus fue del 92\% logrando el 90\% de este valor 23 horas postaplicación mientras que el tiempo en que absorbió la misma proporción de herbicida en $P$. axillaris fue mucho mayor (65 HAT) (Figura 3). Entre distintas especies se pueden encontrar variaciones en cuanto al tiempo de absorción de glifosato. El mismo puede continuar más allá del tercer día post aplicación como en Ipomoea purpurea y Polygonum convolvulus; o no continuar como sucede en Convolvulus arvensis, Convolvulus sepium y Cirsium arvense (Sandberg et al., 1980); con lo cual cada una de las especias estudiadas podría ser ubicada en grupos diferentes.

La translocacion de $\left[{ }^{14} \mathrm{C}\right]$-glifosato se incrementó en ambas especies con el paso del tiempo (Figura 4) siendo Tmax en A. hybridus ssp. hybridus del 64\%, mientras que en $P$. axillaris es significativamente mayor (82\%), mostrando el modelo correlación con los valores medidos (Tabla 3 ). Tanto en A. hybridus ssp. hybridus como en $P$. axillaris el herbicida se movió hacia las demás hojas y hacia el sistema radical (Figura 5). Sin embargo, se observaron algunas diferencias en el patrón de translocación del $\left[{ }^{14} \mathrm{C}\right]$ glifosato. En $P$. axillaris el herbicida radiomarcado se encontró específicamente en las hojas más jóvenes, mientras que en $A$. hybridus ssp. hybridus el patrón de distribución pareciera ser más generalizado, llegando a hojas ya desarrolladas. Un comportamiento similar fue reportado para plantas de Lolium rigidum donde los biotipos sensible y resistentes translocaban igual fracción de herbicida pero el sensible mostraba una mayor acumulación en las bases

Tabla 2 - Parámetros de la ecuación que representa la regresión hiperbólica cuadrática de la absorción de [ $\left.{ }^{14} \mathrm{C}\right]$ glifosato en en A. hybridus ssp. hybridus y Petunia axillaris

\begin{tabular}{|l|c|c|c|}
\hline \multicolumn{1}{|c|}{ Parámetro } & Media (DE) & Valor de $\mathrm{p}$ & Observado \\
\hline Amax: A. hybridus ssp. hybridus & $92,91(1,643)$ & $<0,0001$ & 90,64 \\
\hline Amax:Petunia axillaris & $90,23(3,687)$ & $<0,0001$ & 64,46 \\
\hline t90: A. hybridus ssp. hybridus & $23,69(4,049)$ & $<0,0001$ & 24,00 \\
\hline t90:Petunia axillaris & $65,30(8,454)$ & $<0,0001$ & 72,00 \\
\hline Amax ratio (A. hybridus ssp. hybridus/Petunia axillaris) & $1,02(0,045)$ & 0,5205 & \\
\hline t90 ratio (A. hybridus ssp. hybridus /Petunia axillaris) & $0,36(0,088)$ & $<0,0001$ & \\
\hline
\end{tabular}

Los parámetros Amax y t90 representan el valor máximo de absorción estimado y el tiempo necesario para alcanzar el 90\% de la absorción máxima respectivamente, el valor de p es el nivel de probabilidad de significancia de cada factor para el modelo. 


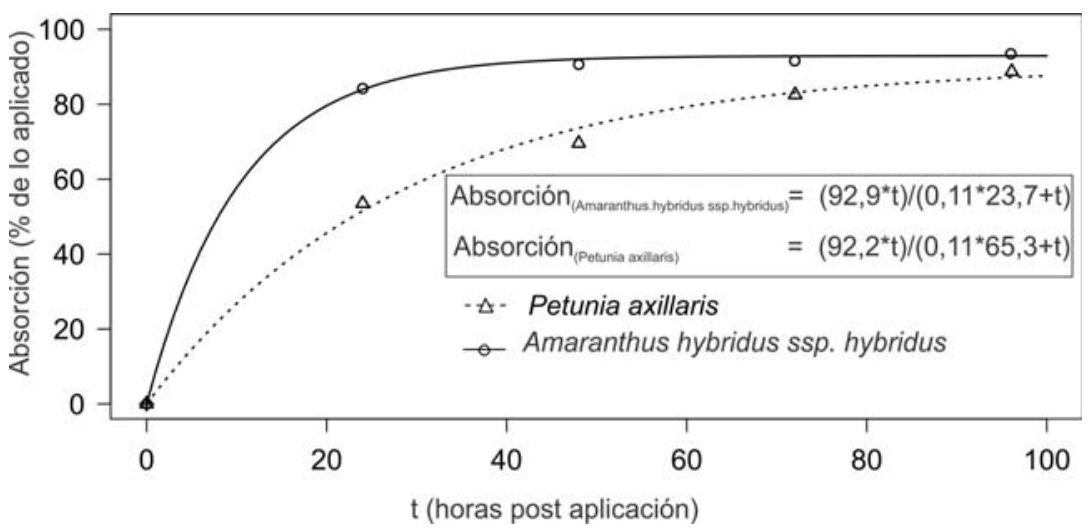

Datos ajustados al modelo hiperbólico cuadrático.

Figura 3 - Absorción de glifosato aplicado en BBCH 1.3 en P. axillaris y A. hybridus ssp. hybridus.

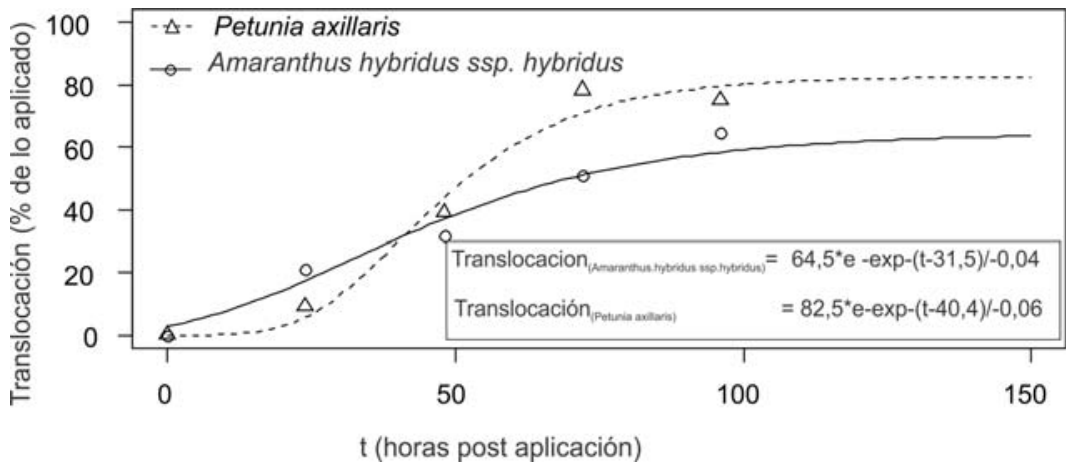

Datos ajustados al modelo Gompertz.

Figura 4 - Translocación de glifosato en P. axillaris y A. hybridus ssp. hybridus.

de las hojas más viejas y raíces mientras que el resistente acumulaba mayor cantidad en los ápices foliares (Lorraine-Colwill et al., 2002). Esta diferencia también es observable externamente a través de la sintomatología que muestra la planta. Al segundo día postaplicación $P$. axilaris mostró una clorosis en la zona más apical del tallo principal, la que al día nueve se extendió a las últimas hojas expandidas, pero sólo en la región basal de las mismas. Mientras tanto las hojas basales no mostraron sintomas. Esta ausencia de sintomatologia en las partes viejas de la planta fue reportada también para biotipos resistentes al glifosato de Conyza bonariensis (Chodová et al., 2009). Además de la clorosis (que fue el sintoma más acentuado) pudieron apreciarse los demás síntomas característicos descritos para plantas tratadas con glifosato, como ser necrosis en las hojas y presencia de color rojizo en el follaje (Tuesca \& Nisensohn, 2004).

Desde el punto de vista estructural las plantas de $P$. axillaris no tratadas con herbicida presentaron un tallo principal, monopodial (sin floración terminal), de más de 15 entrenudos cortos (Figura 6A). La ramificación se inició cuando el meristema terminal del tallo principal cesó temporalmente su crecimiento, luego de producir el nudo 15. El desarrollo de las ramificaciones fue acrópeto y la longitud de las ramas variable según su posición $(5-30 \mathrm{~cm})$, siendo las superiores las más cortas. Estas ramas de primer orden son de crecimiento definido $\mathrm{y}$ 
Tabla 3 - Parámetros estimados de la ecuación gompertz a los que se ajustan los datos de translocación de [ $\left.{ }^{14} \mathrm{C}\right]$ glifosato en A. hybridus ssp. hybridus y Petunia axillaris

\begin{tabular}{|l|c|c|c|}
\hline \multicolumn{1}{|c|}{ Parámetro } & Media (SE) & Valor de p & $\begin{array}{c}\text { Observado } \\
\text { (96 HAT) }\end{array}$ \\
\hline b: A. hybridus ssp. hybridus & $-0,0357(0,0068)$ & $<0,0001$ & - \\
\hline b:Petunia axillaris & $-0,0594(0,0126)$ & $<0,0001$ & - \\
\hline Tmax: A. hybridus ssp. hybridus & $64,4924(3,7306)$ & $<0,0001$ & 64,46 \\
\hline Tmax: Petunia axillaris & $82,5380(4,9046)$ & $<0,0001$ & 75,78 \\
\hline k: A. hybridus ssp. hybridus & $31,4740(4,1767)$ & $<0,0001$ & - \\
\hline k:Petunia axillaris & $40,4115(2,6609)$ & $<0,0001$ & - \\
\hline Tmax (A. hybridus ssp. hybridus /Petunia axillaris) & $0,7814(0,0647)$ & 0,0244 & - \\
\hline
\end{tabular}

Los parámetros c y e no tienen interpretación directa y Tmax representa el valor máximo de translocación, el valor de p es el nivel de probabilidad de significancia de cada factor para el modelo.

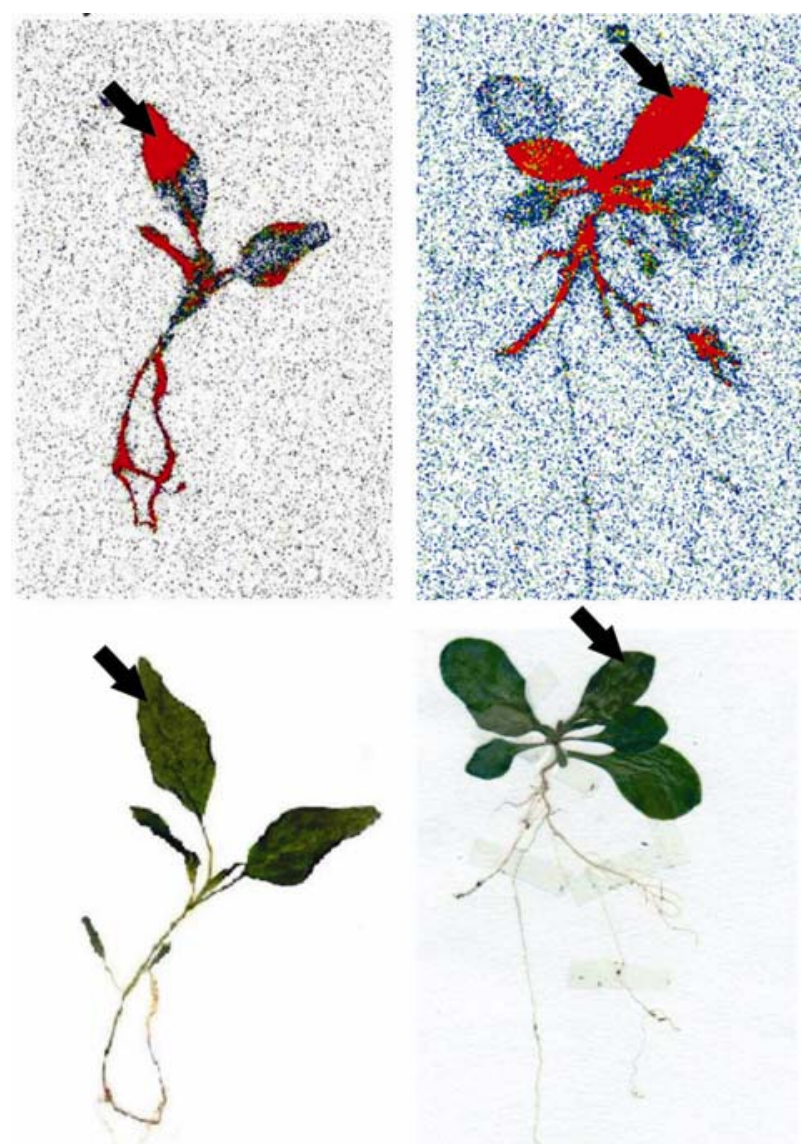

Figura 5 - Observación de $\left[{ }^{14} \mathrm{C}\right]$ glifosato mediante Autoradiografia en $P$. axillaris (derecha) y A. hybridus ssp. hybridus (izquierda), 72 horas post aplicación, las flechas señalan el punto de aplicación del herbicida marcado.

desarrollan un sistema simpodial en el cual la yema terminal remata en una flor, del nudo inmediato inferior se desarrolla una rama de segundo orden que rematará también en flor; y así sucesivamente hasta el tercer orden de ramificación (Figura 6A). Cada planta desarrolla, para el periodo estudiado, alrededor de ocho flores en promedio y siete ramas basales (con un promedio de 27 nudos cada una). Las plantas coleccionada a campo, sin tratamiento, de mayor tamaño que las del ensayo, presentaron la misma estructura que la descrita, mostrando sólo una mayor cantidad de hojas sobre el eje principal (el número máximo encontrado fue de 25 hojas). El largo de las ramas basales también fue mayor, alcanzando más de $40 \mathrm{~cm}$ y la ramificación en las ramas florales llegó a cuarto orden.

El desarrollo y las características de las ramificaciones descritas para las plantas sin tratar permiten establecer tres zonas sobre el eje principal: la primera abarca desde el nudo cotiledonar hasta el nudo dos inclusive, donde las yemas axilares no se desarrollan (zona de inhibición basal); la segunda zona abarca desde el nudo tres hasta el nudo once, al momento de la aplicación, donde las yemas axilares desarrollan acrópetamente sendas ramas (zona de enriquecimiento); y finalmente la zona distal del eje donde al igual que en la zona basal no se produce el desarrollo de ramas (zona de inhibición apical) (Figura 6A). La región correspondiente a la zona de enriquecimiento amplía su tamaño a medida que el eje se desarrolla, manteniendo siempre, sin embargo una zona de inhibición apical compuesta por varios entrenudos.

Cuando se aplica un herbicida se crean condiciones ambientales artificiales extremas negativas para el crecimiento, en 

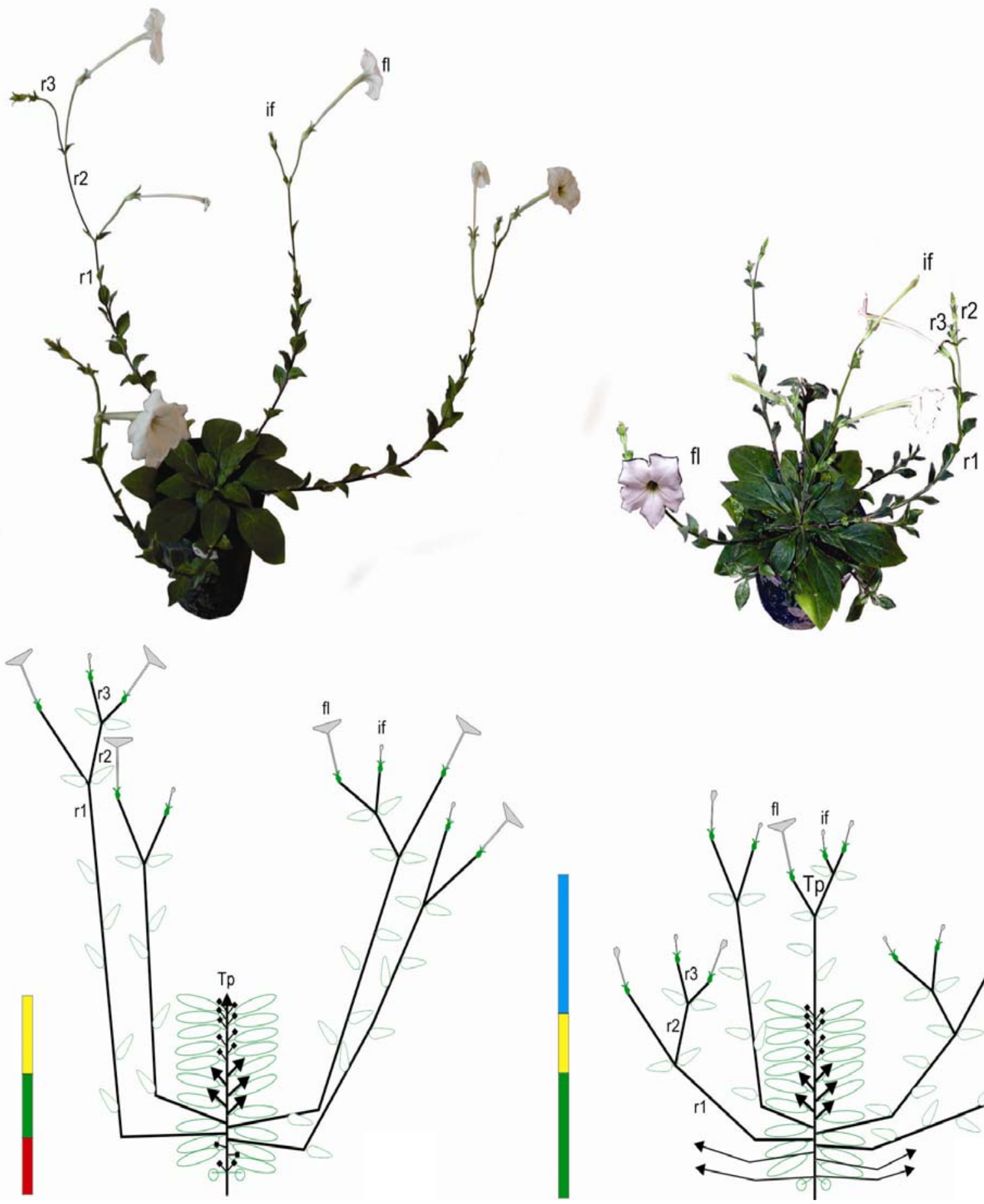

(A)

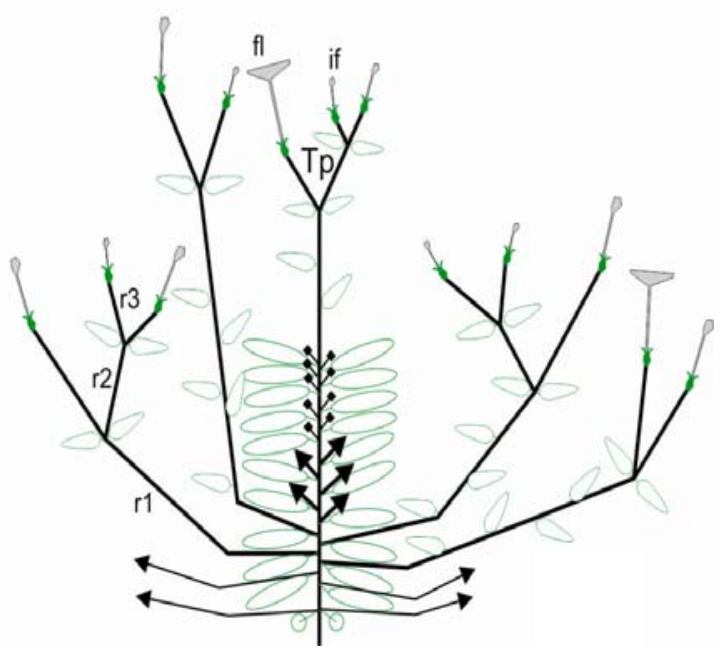

(B)

Referencias: if: primordio floral; fl: flor; r1-3: ramas de primero a tercer orden respectivamente; Tp: tallo principal. La barra señala la zonación del eje principal: rojo, zona de inhibición basal; verde, zona de enriquecimiento; amarillo, zona de inhibición apical, azul, zona de floración terminal.

Figura 6 - Estructura de P. axillaris; (A) imagen (arriba) y esquema (abajo) de planta no tratada; (B) imagen (arriba) y esquema (abajo) de planta con alteraciones producidas por la aplicación de glifosato. 
consecuencia una planta que sobrevive a la acción de un herbicida modifica su comportamiento debido principalmente a la acción ejercida por el herbicida sobre los meristemas (Meusel et al., 1977). La aplicación de glifosato modificó el comportamiento de $P$. axilaris, las plantas tratadas mostraron a los 14 días postaplicación una serie de variaciones que abarcaron diferentes aspectos de la estructura. Sobre el eje principal se observó la elongación de los entrenudos apicales (a partir del 20) y el desarrollo de una flor terminal, con formación de ramas florales cortas sobre el eje principal (desarrollando un sistema simpodial con ramas florales de hasta tercer orden) (Figura 6B). Las yemas axilares ubicadas en la zona de inhibición basal se activaron generando sendas ramas. La cantidad de ramas basales por planta aumentó a 18 (valores promedio), aunque las mismas fueron más cortas, presentando no más de nueve nudos. Este aumento en las ramificaciones en plantas tratadas ha sido reportado en especies perennes que han sobrevivido a la aplicación de herbicidas, con una respuesta típica de producción de ramas a partir de yemas axilares que originalmente se encontraban inhibidas en la forma de crecimiento normal de la especie (Panigo et al., 2012). Este fenómeno podria explicarse debido a que el glifosato se transloca hacia las zonas en activo crecimiento de la planta (Shaner, 2009) y no hacia las regiones de actividad inhibida. En el momento de la aplicación $P$. axillaris se encontraba formando las ramas basales, las que retrasaron su desarrollo y consecuentemente su floración. Por tanto, las plantas tratadas presentaron además un número menor de flores al finalizar el ensayo. Sin embargo, esto también produjo un aumento de la cantidad de entrenudos producidos antes de iniciar la floración, lo que determinó una mayor disponibilidad de yemas axilares.

Estas variaciones estructurales postaplicación afectaron la zonación del eje principal de las plantas tratadas que en consecuencia carece de zona de inhibición basal, mostrando una amplia zona de producción de ramas basales (zona de enriquecimiento), seguida por una zona de inhibición apical (compuesta por dos entrenudos cortos distales) y finalmente la zona distal donde se desarrolla una región de floración apical (Figure 6B). La nueva región de producción de ramas florales sobre el eje principal puede ser considerada como una amplificación de la zona de enriquecimiento. En esta zona (al igual que la de enriquecimiento basal) las yemas latentes brotan facultativamente, fuertemente influenciadas por variables ambientales (Rua \& Weberling, 1998) y tiene la función de aumentar la producción de estructuras reproductivas, aumentando consecuentemente el número de semillas producidas.

El glifosato es un herbicida que afecta principalmente zonas meristemáticas (Shaner, 2009), sin embargo, la posibilidad y lugar dónde se activará una yema axilar involucra la integración de un amplio rango de factores externos (ambientales) e internos (fisiológicos y de desarrollo) (Leyser, 2009). Esto último hace que la disponibilidad de meristemas activos varie constantemente durante el desarrollo de planta afectando el comportamiento y respuesta a distintos factores en función del momento de la aplicación. La planta construye su forma a medida que avanza el desarrollo y se establecen las ramificaciones (Barthélémy \& Caraglio, 2007), por lo que la magnitud de la recuperación en plantas tolerantes estará afectada fuertemente por el estadio de desarrollo al cuál la misma es tratada.

Las especie $P$. axillaris mostró una sensibilidad menor al glifosato, una menor retención foliar, mayor tiempo de absorción y un patrón diferente de translocación con relación a la especie utilizada como patrón sensible, sin mostrar inhibición de la ruta metabólica del ácido shinkímico; pudiendo por lo tanto catalogarse como especie tolerante a glifosato.

En $P$. axillaris se observa claramente una combinación de características morfológicas, estructurales y fisiológicas que determinan su ventaja adaptativa para permanecer y aumentar su población en sistemas con uso intensivo de glifosato. La respuesta morfológica observada para $P$. axillaris no podría ser catalogada como estrategia de escape, ya que el herbicida afecta cuantitativamente la estructura, sin embargo, los niveles no son suficientes como para impedir la reproducción y el desarrollo de los ejemplares, posibilitando su persistencia en el sistema. 


\section{AGRADECIMIENTOS}

Este trabajo fue realizado parcialmente con fondos provenientes de los proyectos $\mathrm{CAI}+\mathrm{D}$ UNL y PIP- CONICET.

\section{LITERATURA CITADA}

BAERSON, S. R. et al. Glyphosate-Resistant Goosegrass. Identification of a mutation in the Target Enzyme 5Enolpyruvylshikimate-3-Phosphate Synthase. Plant Physiol., v. 129, n. 3, p. 1265-1275, 2002.

BARTHÉLÉMY, D.; CARAGLIO, Y. Plant architecture: A dynamic, multilevel and comprehensive approach to plant form, structure and ontogeny. Ann. Bot., v. 99, n. 3, p. 375-407, 2007.

BARTHÉLÉMY, D. et al. Architectural concepts for tropical trees. In: Tropical Forests: botanical dynamics, speciation and diversity. London: Academic Press, 1989. 100 p.

BINKHOLDER, K. M. et al. Selection of glyphosateresistant annual bluegrass (poa annua) on a golf course. Weed Sci., v. 59, n. 3, p. 286-289, 2011.

CHODOVÁ, D. et al. Horseweed with Reduced Susceptibility to Glyphosate Found in the Czech Republic. J. Agric. Food Chem., v. 57, n. 15, p. 6957-6961, 2009.

CHRISTOFFOLETI, P. J. et al. Glyphosate sustainability in South American cropping systems. Pest Manage. Sci., v. 64, n. 4, p. 422-427, 2008.

CRUZ-HIPOLITO, H. et al. Glyphosate tolerance by Clitoria ternatea and Neonotonia wightii plants involves differential absorption and translocation of the herbicide. Plant Soil, v. 347, n. 1-2, p. 221-230, 2011.

DELLAFERRERA, I. et al. Relevamiento de malezas en cultivos de soja en sistema de siembra directa con glifosato del departamento San Justo (provincia de Santa Fe). R. FAVE Ci Agr., v. 5/6, n. 1, p. 15-25, 2007.

FACCINI, D.; PURICELLI, E. Eficacia de herbicidas según la dosis y el estado de crecimiento de malezas presentes en un suelo en barbecho. Agriscientia, v. 24, n. 1, p. 29-35, 2007.

FENG, P. C. C. et al. Investigations into glyphosate-resistant horseweed (Conyza canadensis): retention, uptake, translocation, and metabolism. Weed Sci., v. 52, n. 4, p. 498-505, 2004.

GAINES, T. A. et al. Mechanism of Resistance of Evolved Glyphosate-Resistant Palmer Amaranth (Amaranthus palmeri). J. Agric. Food Chem., v. 59, n. 11, p. 5886-5889, 2011.
GALON, L. et al. Glyphosate translocation in herbicide tolerant plants. Planta Daninha, v. 31, n. 3, p. 193-201, 2013.

GAUVRIT, C. Glyphosate response to calcium, ethoxylated amine surfactant, and ammonium sulfate. Weed Technol., v. 17, n. 4, p. 799-804, 2003.

GE, X. et al. Rapid vacuolar sequestration: the horseweed glyphosate resistance mechanism. Pestic. Manage. Sci., v. 66, n. 4, p. 345-348, 2010.

GONZAiLEZ-TORRALVA, F. et al. Differential susceptibility to glyphosate among the Conyza weed species in Spain. J. Agric. Food Chem., v. 58, n. 7, p. 4361-4366, 2010.

HESS, M. et al. Use of the extended BBCH scale - general for the descriptions of the growth stages of mono; and dicotyledonous weed species. Weed Res., v. 37, n. 6, p. 433-441, 1997.

JOHNSON, W. G. et al. Influence of glyphosate-resistant cropping systems on weed species shifts and glyphosateresistant weed populations. Eur. J. Agron., v. 31, n. 3, p. 162-172, 2009.

KNISS, A. R. et al. Nonlinear regression analysis of herbicide absorption studies. Weed Sci., v. 59, n. 4, p. 601-610, 2011.

LEYSER, O. The control of shoot branching: an example of plant information processing. Plant Cell Environ., v. 32, n. 6, p. 694-703, 2009.

LORRAINE-COLWILL, D. et al. Investigations into the mechanism of glyphosate resistance in Lolium rigidum.

Pestic. Biochem. Physiol., v. 74, n. 1, p. 62-72, 2002.

MALPASSI, R. Efecto de la aplicación de herbicidas sobre la morfología y anatomía de Eleusine indica (L.)

Gaertner y Portulaca oleracea L. Tesis (Doctorado) Universidad Nacional de Río Cuarto, Rio Cuarto, 2004.

MELLENDORF, T. G. et al. Influence of plant height and glyphosate on saflufenacil efficacy on glyphosate-resistant horseweed (Conyza canadensis). Weed Technol., v. 27, n. 3, p. 463-467, 2013.

MEUSEL, M. E. et al. The study of growth-forms of higher plants in relation to application of biocide. In: Miyawaki, A., Tüxen, R. (Eds.), Vegetation Science and Environmental Protection I. Maruzen, Tokyo, 1977. p. 576

OWEN, M. D. Weed species shifts in glyphosate-resistant crops. Pest Manage. Sci., v. 37, n. 4, p. 377-387, 2008.

PANIGO, E. S. et al. Glyphosate-induced structural variations in Commelina erecta L. (Commelinaceae). Ecotoxicol. Environ. Safety, v. 76, n. 1, p. 135-142, 2012. 
POWLES, S. B.; YU, Q. Evolution in action: plants resistant to herbicides. Ann. Rev. Plant Biol., v. 61, n. 1, p. 317-347, 2010.

PURICELLI, E.; PAPA, J. C. Growth of Parietaria debilis in fallow and in undisturbed areas. Weed Res., v. 46, n. 1, p. 129137, 2006.

RITZ, C.; STREIBIG, J. C. Bioassay analysis using R. J. Stat. Software, v. 12, n. 1, p. 1-22, 2005.

ROJANO-DELGADO, A. M. et al. Limited uptake, translocation and enhanced metabolic degradation contribute to glyphosate tolerance in Mucuna pruriens var. utilis plants. Phytochemistry, v. 73, n. 1, p. 34-41, 2012.

RUA, G.; WEBERLING, F. Growth form and inflorescence structure of Paspalum L. (Poaceae, Paniceae): a comparative morphological approach. Beiträge Biol. Pflanzen, v. 69, n. 3, p. 363-431, 1998.

SANDBERG, C. L. et al. Absorption, translocation and metabolism of 14C-glyphosate in several weed species. Weed Res., v. 20, n. 1, p. 195-200, 1980.

SCURSONI, J. A.; SATORRE, E. H. Glyphosate management strategies, weed diversity and soybean yield in Argentina. Crop Protec., v. 29, n. 9, p. 957-962, 2010.
SHANER, D. L. Role of translocation as a mechanism of resistance to glyphosate. Weed Sci., v. 57, n. 1, p. 118-123, 2009.

SINGH, B.; SHANER, D. Rapid determination of glyphosate injury to plants and identification of glyphosateresistant plants. Weed Technol., v. 12, n. 3, p. 527-530, 1998.

STREIBIG, J. C.; KUDSK, P. Herbicide bioassays. Boca Raton: CRC Press, 1993. p.294

TREJO, D.; SOBRERO, M. Determinacion de la eficacia de glifosato para el control de Petunia axillaris (Lam.) Britton, Stern \& Poggenb. In: CONGRESSO BRASILEIRO DA CIÊNCIA DAS PLANTAS DANINHAS, 28., 2012, Campo Grande. Actas... Campo Grande: 2012.

TUESCA, D.; NISENSOHN, L. Inhibidores de las síntesis de aminoácidos. In: Vitta, J., (Ed.). Herbicidas: características y fundamentos de su actividad. Rosario: Universidad Nacional de Rosario, 2004. p. 83.

VELINI, E. D. et al. Glyphosate applied at low doses can stimulate plant growth. Pest Manage. Sci., v. 64, n. 2, p. 489-496, 2008. 